\title{
ON THE UNIQUENESS OF GIBBS STATES IN THE PIROGOV-SINAI THEORY
}

\author{
AZER KERIMOV \\ Department of Mathematics, Bilkent University, \\ 06533 Bilkent, Ankara, Turkey \\ kerimov@fen.bilkent.edu.tr
}

Received 16 September 2005

\begin{abstract}
We consider models of classical statistical mechanics satisfying natural stability conditions: a finite spin space, translation-periodic finite potential of finite range, a finite number of ground states meeting Peierls or Gertzik-Pirogov-Sinai condition. The Pirogov-Sinai theory describes the phase diagrams of these models at low temperature regimes. By using the method of doubling and mixing of partition functions we give an alternative elementary proof of the uniqueness of limiting Gibbs states at low temperatures in ground state uniqueness region.
\end{abstract}

Keywords: Ground state; partition function; Gibbs state; extreme Gibbs state; Peierls condition; Pirogov-Sinai theory; contour model.

\section{Introduction}

Pirogov-Sinai theory ${ }^{1-3}$ investigates the phase diagrams of low temperature spin models of statistical mechanics. Roughly speaking, the theory taking its origins from fundamental work of Peierls, ${ }^{4}$ states that the qualitative picture at temperature zero remains valid at any sufficiently low temperature. The problem of the completeness of the phase diagram, that is, whether the theory provides all extreme periodic Gibbs states constructed in this theory has attracted the interest of many authors. Zahradnik ${ }^{5}$ proved that the Gibbs states constructed in Pirogov-Sinai theory are the only extreme and translation-periodic Gibbs states. Alternative proofs of the uniqueness of the Gibbs states at low temperatures in the special case of uniqueness of the ground state were independently obtained in Refs. 6-8. In Pirogov-Sinai theory, in the regions where there is a unique translation-periodic Gibbs state there are no other (translation-periodic or non translation-periodic) Gibbs states. ${ }^{9}$ In this paper we give one more alternative simple proof of the uniqueness.

In this paper we investigate classical models in the classical settings: a finite spin space, translation-periodic finite potential of finite range, a finite number of ground states and their stability (so-called Peierls or Gertzik-Pirogov-Sinai condition). We give a new proof of the uniqueness of Gibbs states at law temperatures in the one- 
ground-state region: there is no any translation-periodic or non translation-periodic Gibbs state except constructed one in the Pirogov-Sinai theory. The proof is based on the verification of non-singularity of two arbitrary extreme Gibbs states. ${ }^{10}$ The main method of this tricky proof is a "coupling" and "mixing" of corresponding partition functions. Considering two (here independent) realizations of Gibbs states means that we are employing a (here product) coupling argument. Such coupling arguments are also at the origin of the disagreement percolation approach to prove uniqueness of Gibbs states. ${ }^{11,12}$ The proof also gives a simple explanation of the uniqueness.

\section{Formulations}

Let $\mathbf{Z}^{\nu}$ be the $\nu$-dimensional cubic lattice. The spin variables $\phi(x)$ associated with the lattice sites $x$ take values from the finite set $\Phi=\{1,2, \ldots, r\}$.

Consider a model on $\mathbf{Z}^{\nu}$ with the formal Hamiltonian

$$
H_{0}(\phi)=\sum_{A \subset \mathbf{Z}^{\nu}} U_{0}\left(\phi_{A}\right)
$$

where $\phi_{A}$ is the restriction of the configuration $\phi \in \Phi^{\mathbf{Z}^{\nu}}$ to the set $A \subset \mathbf{Z}^{\nu}$, the potential $U_{0}\left(\phi_{A}\right): \Phi^{A} \rightarrow \mathbf{R}$ is of finite range $R: U_{0}\left(\phi_{A}\right)=0$ if the diameter of $A$ exceeds $R$ and translation periodic: $U_{0}\left(\phi_{A}\right)=U_{0}\left(\phi_{A+t}\right)$ for any $t$ from some subgroup of $\mathbf{Z}^{\nu}$ of finite index.

We say that a configuration $\phi$ is a ground state of the model (1) if $H_{0}\left(\phi^{\prime}\right)-$ $H_{0}(\phi) \geq 0$ for any finite perturbation $\phi^{\prime}$ of $\phi$ (the set $\left\{x: \phi_{x} \neq \phi_{x}^{\prime}\right\}$ is finite).

We suppose that the model (1) has a finite number of ground states invariant under the action of some subgroup of $\mathbf{Z}^{\nu}$ of finite index. Later on, without loss of generality, we will suppose that the potential of the model (1) is translationinvariant and translation-periodic ground states of (1) are translation-invariant. Indeed, one can partition the lattice into disjoint cubes $Q(z)$ centered at $z \in q \mathbf{Z}^{\nu}$ with an appropriate value of $q$ and replace the spin space from $\Phi$ to $\Phi^{Q}$. If we choose $q$ exceeding the interaction radius $R$ the model (1) becomes a model with the nearest neighbor and diagonal interaction.

Let $V \subset \mathbf{Z}^{\nu}$ be a finite domain and $\bar{\phi}_{V^{c}}$ be boundary conditions given on its complement $V^{c}=\mathbf{Z}^{\nu}-V$. The conditional Hamiltonian is defined as

$$
H_{0}\left(\phi_{V} \mid \bar{\phi}_{V^{c}}\right)=\sum_{A \subset \mathbf{Z}^{\nu}: A \cap V \neq \emptyset} U_{0}\left(\phi_{A}\right)
$$

where $\phi_{A}$ is a concatenation of the configurations $\phi_{V}$ and $\bar{\phi}_{V^{c}}$ on $A: \phi_{A}=\phi_{A \cap V}+$ $\bar{\phi}_{A \cap V^{c}}$, i.e. the spin at site $x$ is $\phi_{x}$ if $x \in A \cap V$ and $\bar{\phi}_{x}$ if $x \in A \cap V^{c}$.

Without loss of generality, the translation-invariant ground states of the model (1) we denote by $\phi^{(1)}, \ldots, \phi^{(m)}$ and suppose that $\phi_{x}^{(k)}=k$ for each $x \in \mathbf{Z}^{\nu}$.

For a fixed configuration $\phi$ in $\mathbf{Z}^{\nu}$ we say that a lattice cube $Q_{2}(x)$ of linear size 2 centered at lattice point $x$ is not regular if $\phi_{Q_{2}(x)} \neq \phi_{Q_{2}(x)}^{(k)}$ for each $k=1,2, \ldots, m$. Two non-regular cubes are called connected provided their intersection is not empty. 
The connected components of non-regular cubes are called supports of contours and are denoted by $\operatorname{supp}(\gamma)$. A pair $(\operatorname{supp}(\gamma), \phi(\operatorname{supp}(\gamma)))$ is called a contour and will be denoted by $\gamma$.

Consider a configuration $\phi_{V}$ at fixed boundary conditions $\phi^{(k)}$. The expression

$$
H_{0}\left(\phi_{V} \mid \phi_{V^{c}}^{(k)}\right)-H_{0}\left(\phi_{V}^{(k)} \mid \phi_{V^{c}}^{(k)}\right)
$$

expressing the energy difference between $\phi_{V}$ and the ground state $\phi^{(k)}$ can be written as $^{3}$

$$
\sum_{\gamma_{i}} \sum_{A \cap \operatorname{supp}\left(\gamma_{i}\right) \neq \emptyset}\left(U_{0}\left(\phi_{A}\right)-U_{0}\left(\phi_{A}^{(k)}\right)\right) .
$$

The last expression shows that the energy difference is concentrated on nonregular cubes. We suppose that the energy excess is proportional to the total volume of non-regular cubes, namely for each ground state $\phi^{(k)}$ the Hamiltonian (1) satisfies the well-known Peierls condition:

$$
\sum_{A \cap \operatorname{supp}\left(\gamma_{i}\right) \neq \emptyset}\left(U_{0}\left(\phi_{A}\right)-U_{0}\left(\phi_{A}^{(k)}\right)\right) \geq \tau\left|\gamma_{i}\right|
$$

where $\tau$ is a positive absolute constant and $\left|\gamma_{i}\right|$ denotes the number of sites in $\operatorname{supp}\left(\gamma_{i}\right)$. In this case the ground states $\phi^{(k)}$ are called stable ground states.

Suppose that a vector $\lambda=\left(\lambda_{1}, \ldots, \lambda_{m-1}\right)$ belongs to some open neighborhood of the origin in $\mathbf{R}^{m-1}$. We define a perturbed formal Hamiltonian

$$
H(\phi)=H_{0}(\phi)+\sum_{n=1}^{m-1} \lambda_{n} H_{n}(\phi)
$$

where Hamiltonians $H_{n}(\phi)=\sum U_{n}(\phi), n=1, \ldots, m-1$ share all conditions with $H_{0}$. We also suppose that this perturbation removes the degeneracy of the ground state. $^{3}$

The finite-volume Gibbs distribution corresponding to the boundary conditions $\bar{\phi}_{V^{c}}$ is

$$
\mu_{V, \bar{\phi}_{V^{c}}}\left(\phi_{V}\right)=\frac{\exp \left(-\beta H\left(\phi_{V} \mid \bar{\phi}_{V^{c}}\right)\right)}{\Xi\left(V, \bar{\phi}_{V^{c}}\right)}
$$

where $\beta$ is the inverse temperature, the conditional Hamiltonian $\left.H\left(\phi_{V} \mid \bar{\phi}_{V^{c}}\right)\right)=$ $\sum_{A \cap V \neq \emptyset} \sum_{n=0}^{m-1} \lambda_{n} U_{n}\left(\phi_{A}\right)$ and the partition function $\Xi\left(V, \bar{\phi}_{V^{c}}\right)=\sum_{\phi_{V}} \times$ $\exp \left(-\beta H\left(\phi_{V} \mid \bar{\phi}_{V^{c}}\right)\right) \cdot \mu_{V, \phi_{V^{c}}^{(k)}}(\cdot)$ will be denoted by $\mu_{V}^{(k)}(\cdot)$ below.

Theorem 1.1,3 Consider a model with the Hamiltonian (3) at some fixed value of the vector $\lambda=\left(\lambda_{1}, \ldots, \lambda_{m-1}\right)$ and suppose that $\phi^{(k)}$ is an arbitrary stable ground state of the perturbed Hamiltonian (3). Then there exists a value of the inverse temperature $\beta_{0}(\lambda)$ such that for all $\beta>\beta_{0}(\lambda)$ ground state $\phi^{(k)}$ generates a translationinvariant Gibbs state 1,3 $^{1}$

$$
\mu^{(k)}(\cdot)=\lim _{V \rightarrow \mathbf{Z}^{\nu}} \mu_{V}^{(k)}(\cdot) .
$$

These Gibbs states are different for different values of $k$. 
Theorem 2. ${ }^{5}$ Consider a model with the Hamiltonian (3) at some fixed value of the vector $\lambda=\left(\lambda_{1}, \ldots, \lambda_{m-1}\right)$. There exists a value of the inverse temperature $\beta_{0}(\lambda)$ such that for all $\beta>\beta_{0}(\lambda)$ Gibbs states constructed in Theorem 1 are the only translation-periodic Gibbs states of the model (3).

In the special case when the model (3) has a unique ground state we get the following:

Corollary ${ }^{6-8}$ Let $\lambda$ be a value such that the model (3) has a unique stable ground state. There exists a value of the inverse temperature $\beta_{0}(\lambda)$ such that for all $\beta>$ $\beta_{0}(\lambda)$ the model (3) has a unique translation-periodic Gibbs state.

The statement of this Corollary can be slightly improved:

Theorem 3. ${ }^{9}$ Let $\lambda$ be a value such that the model (3) has a unique (periodic or non-periodic) stable ground state. There exists a value of the inverse temperature $\beta_{0}(\lambda)$ such that for all $\beta>\beta_{0}(\lambda)$ the model (3) has a unique Gibbs state.

As it was mentioned in the introduction the proof of the Corollary was given in Refs. 5-8 by different authors and different methods. Its extension Theorem 3 has a proof based on the method of polymer expansions. ${ }^{9}$

In the present paper we give an alternative elementary proof of Theorem 3 based on the following tricky idea: instead of a probability space we consider two probability spaces and after that we "mix" these spaces in convenient way. The proof also gives descriptive and clear explanation of uniqueness.

\section{Proofs}

Let $\mu^{1}$ and $\mu^{2}$ be two extreme Gibbs states corresponding to arbitrary fixed boundary conditions $\phi^{1}$ and $\phi^{2}$ (not necessarily ground states, note that $\phi^{(k)}$ denotes a ground state, but $\phi^{k}$ denotes an arbitrary configuration). It is well known that $\mu^{1}$ and $\mu^{2}$ are singular or coincide. ${ }^{3,10}$ We prove the uniqueness of the Gibbs states of the model (3) by showing that $\mu^{1}$ and $\mu^{2}$ are not singular and therefore coincide.

Let $V_{N}$ be a $\nu$-cube with the center at the origin and with the length of edge $2 N: V_{N}=\left\{x_{1}, x_{2}, \ldots, x_{\nu}:-N \leq x_{i} \leq N, i=1,2, \ldots, \nu\right\}$.

Take $M<N$ and let $\mu_{V_{N}}^{k}\left(\phi_{V_{M}}^{\prime}\right)$ be the probability of the event that the restriction of the configuration $\phi_{V_{N}}$ to $V_{M}$ coincides with $\phi_{V_{M}}^{\prime}$. Theorem 3 is a direct consequence of the following.

Theorem 4. Let $\lambda$ be a value such that the model (3) has a unique ground state $\phi^{(p)}$. There exists a value of the inverse temperature $\beta_{0}(\lambda)$ such that for all $\beta>$ $\beta_{0}(\lambda)$ Gibbs states $\mu^{1}$ and $\mu^{2}$ are not singular.

Proof. In order to show that extreme Gibbs states $\mu^{1}$ and $\mu^{2}$ are not singular, we prove that there exist two positive constants $c_{1}$ and $c_{2}$ such that for any $M$ 
and $\phi_{V_{M}}^{\prime}$

$$
c_{1} \leq \mu^{1}\left(\phi_{V_{M}}^{\prime}\right) / \mu^{2}\left(\phi_{V_{M}}^{\prime}\right) \leq c_{2} .
$$

Since Gibbs states $\mu^{1}$ and $\mu^{2}$ are weak limits of finite volume Gibbs states $\mu_{V}^{1}$ and $\mu_{V}^{2}$ (corresponding to arbitrary boundary conditions $\phi^{1}$ and $\phi^{2}$ ) when $N \rightarrow \infty$, for establishing the inequality (5) we prove that there exist two positive constants $c_{1}$ and $c_{2}$ such that for arbitrary boundary conditions $\phi^{1}$ and $\phi^{2}$ and for any $M$ and $\phi_{V_{M}}^{\prime}$ there exists a number $N_{0}(M)$ such that for any $N>N_{0}(M)$

$$
c_{1}<\mu_{V_{N}}^{1}\left(\phi_{V_{M}}^{\prime}\right) / \mu_{V_{N}}^{2}\left(\phi_{V_{M}}^{\prime}\right)<c_{2} .
$$

Consider the $\mu_{V_{N}}^{k}(k=1,2)$ probability of the event that the restriction of the configuration $\phi_{V_{N}}$ to $V_{M}$ coincides with $\phi_{V_{M}}^{\prime}$ :

$$
\begin{aligned}
\mu_{V_{N}}^{k}\left(\phi^{\prime}\left(V_{M}\right)\right) & =\frac{\sum_{\phi_{V_{N}}: \phi_{V_{M}}=\phi_{V_{M}}^{\prime}} \exp \left(-\beta H\left(\phi_{V_{N}} \mid \phi_{V_{N}^{c}}^{k}\right)\right)}{\sum_{\phi_{V_{N}}} \exp \left(-\beta H\left(\phi_{V_{N}} \mid \phi_{V_{N}^{c}}^{k}\right)\right)} \\
& =\frac{\exp \left(-\beta H^{i n}\left(\phi_{V_{M}}^{\prime}\right)\right) \Xi\left(V_{N}-V_{M} \mid \phi^{k}, \phi_{V_{M}}^{\prime}\right)}{\sum_{\phi_{V_{M}}^{\prime \prime}} \exp \left(-\beta H^{i n}\left(\phi_{V_{M}}^{\prime \prime}\right)\right) \Xi\left(V_{N}-V_{M} \mid \phi^{k}, \phi_{V_{M}}^{\prime \prime}\right)}
\end{aligned}
$$

where the summation in $\sum_{\phi^{\prime \prime}\left(V_{M}\right)}$ has taken over all possible configurations $\phi^{\prime \prime}\left(V_{M}\right)$, $H^{i n}\left(\phi_{V_{M}}^{\prime}\right)=\sum_{B \subset V_{M}} U\left(\phi^{\prime}(B)\right)$ and $H^{i n}\left(\phi_{V_{M}}^{\prime \prime}\right)=\sum_{B \subset V_{M}} U\left(\phi^{\prime \prime}(B)\right)$ are interior energies of $\phi^{\prime}\left(V_{M}\right)$ and $\phi^{\prime \prime}\left(V_{M}\right) ; \Xi\left(V_{N}-V_{M} \mid \phi^{(k)}, \phi_{V_{M}}^{\prime}\right)$ is a partition function corresponding to the boundary conditions $\phi_{\mathbf{Z}^{\nu}-V_{N}}^{k}, \phi_{V_{M}}^{\prime}$ and $\Xi\left(V_{N}-V_{M} \mid \phi^{k}, \phi_{V_{M}}^{\prime \prime}\right)$ is a partition function corresponding to the boundary conditions $\phi_{\mathbf{Z}^{\nu}-V_{N}}^{(k)}, \phi_{V_{M}}^{\prime \prime}$. The partition functions $\Xi\left(V_{N}-V_{M} \mid \phi^{k}, \phi_{V_{M}}^{\prime}\right)$ and $\Xi\left(V_{N}-V_{M} \mid \phi^{k}, \phi_{V_{M}}^{\prime \prime}\right)$ are denoted correspondingly by $\Xi^{\phi^{k}, \phi^{\prime}}$ and $\Xi^{\phi^{k}, \phi^{\prime \prime}}$ below.

Now we have

$$
\begin{aligned}
\frac{\mu_{V_{N}}^{(1)}\left(\phi_{V_{M}}^{\prime}\right)}{\mu_{V_{N}}^{(2)}\left(\phi_{V_{M}}^{\prime}\right)}= & \frac{\exp \left(-\beta H^{i n}\left(\phi_{V_{M}}^{\prime}\right)\right) \Xi^{\phi^{1}, \phi^{\prime}}}{\sum_{\phi_{V_{M}}^{\prime \prime}} \exp \left(-\beta H^{i n}\left(\phi_{V_{M}}^{\prime \prime}\right)\right) \Xi^{\phi^{1}, \phi^{\prime \prime}}} \frac{\sum_{\phi_{V_{M}}^{\prime \prime}} \exp \left(-\beta H^{i n}\left(\phi_{V_{M}}^{\prime \prime}\right)\right) \Xi^{\phi^{2}, \phi^{\prime \prime}}}{\exp \left(-\beta H^{i n}\left(\phi_{V_{M}}^{\prime}\right)\right) \Xi^{\phi^{2}, \phi^{\prime}}} \\
= & \frac{\Xi^{\phi^{1}, \phi^{\prime}} \sum_{\phi_{V_{M}}^{\prime \prime}} \exp \left(-\beta H^{i n}\left(\phi_{V_{M}}^{\prime \prime}\right)\right) \Xi^{\phi^{2}, \phi^{\prime \prime}}}{\Xi^{\phi^{2}, \phi^{\prime}} \sum_{\phi_{V_{M}}^{\prime \prime}} \exp \left(-\beta H^{i n}\left(\phi_{V_{M}}^{\prime \prime}\right)\right) \Xi^{\phi^{1}, \phi^{\prime \prime}}} .
\end{aligned}
$$

Note that since the quotient of $\left(\sum_{i=1}^{n} a_{i}\right) /\left(\sum_{i=1}^{n} b_{i}\right)$ lies between $\min \left(a_{i} / b_{i}\right)$ and $\max \left(a_{i} / b_{i}\right)$, in order to prove the inequality (6) it is enough to establish the following inequality:

$$
c_{1}<\frac{\Xi^{\phi^{1}, \phi^{\prime}}}{\Xi^{\phi^{2}, \phi^{\prime}}} \frac{\Xi^{\phi^{2}, \phi^{\prime \prime}}}{\Xi^{\phi^{1}, \phi^{\prime \prime}}}<c_{2}
$$

for arbitrary configuration $\phi_{V_{M}}^{\prime \prime}$. 
Let us define a configuration $\phi_{V_{N}-V_{M}}^{\min , k}$ with the minimal energy at fixed $V_{N}$ and boundary conditions $\phi_{\mathbf{Z}^{\nu}-V_{N}}^{k}, \phi_{V_{M}}^{\prime}$ :

$$
H\left(\phi_{V_{N}-V_{M}}^{\min , k} \mid \phi_{\mathbf{Z}^{\nu}-V_{N}}^{k}, \phi_{V_{M}}^{\prime}\right)=\min _{\phi_{V_{N}-V_{M}}} H\left(\phi_{V_{N}-V_{M}} \mid \phi_{\mathbf{Z}^{\nu}-V_{N}}^{k}, \phi_{V_{M}}^{\prime}\right) .
$$

The following simple and natural lemma describes the structure of the configuration $\phi_{V_{N}-V_{M}}^{\min , k}$.

Lemma 1. Let $\lambda$ be a value such that the model (3) has a unique ground state $\phi^{(p)}$. There exists a positive constant $L_{b}$ such that for arbitrary $M, N$ and arbitrary boundary conditions $\phi_{\mathbf{Z}^{\nu}-V_{N}}^{k}, \phi_{V_{M}}^{\prime}$ the restriction of the configuration $\phi_{V_{N}-V_{M}}^{\min , k}$ to the set $V_{N-L_{b}}-V_{M+L_{b}}$ coincides with the ground state $\phi^{(p)}$.

Proof. For each boundary condition $\phi_{\mathbf{Z}^{\nu}-V_{N}}^{k}, \phi_{V_{M}}^{\prime}$ define the value of $L_{b}\left(\mathbf{Z}^{\nu}-V_{N}, \phi_{V_{M}}^{\prime}\right)$. If contrary to the statement of the lemma $L_{b}\left(\mathbf{Z}^{\nu}-V_{N}, \phi_{V_{M}}^{\prime}\right)$ is not bounded uniformly with respect to all $M, N$ and boundary conditions, then there exists a sequence of boundary conditions $\left\{\phi_{\mathbf{Z}^{\nu}-V_{N_{i}}}^{k_{i}}, \phi_{V_{M_{i}}}^{\prime} ; i=1,2, \ldots\right\}$ such that the corresponding sequence $\left\{L_{b}\left(\mathbf{Z}^{\nu}-V_{N_{i}}, \phi_{V_{M_{i}}}^{\prime}\right) ; i=1,2, \ldots\right\}$ is unbounded. This in turn means that in the corresponding sequence of configurations with minimal energy $\phi_{V_{N_{i}}-V_{M_{i}}}^{\min , k_{i}}$ differs from the ground state in unboundedly growing by $i$ area. We can shift the configuration $\phi_{V_{N_{i}}-V_{M_{i}}}^{\min , k_{i}}$ such that this non-regular area will cover the origin and will grow by $i$ in all directions. Let the configuration $\phi^{\text {min }}$ be a limit point of the sequence of these shifted configurations. By construction this configuration is not a ground state $\phi^{(p)}$. On the other hand, let us show that the configuration $\phi^{\text {min }}$ as a limit point of configurations with minimal energy is a ground state $\phi^{(p)}$. Indeed, suppose that $\bar{\phi}^{\text {min }}$ is an arbitrary perturbation of $\phi^{\text {min }}$ on some finite set. Then when $V_{N}$ is sufficiently large (as it is noted in Sec. 2 the interaction potential without loss of generality supposed to be translation-invariant)

$$
\begin{aligned}
H\left(\bar{\phi}^{\min }\right)-H\left(\phi^{\min }\right)= & H\left(\bar{\phi}_{V_{N}-V_{M}}^{\min , k} \mid \phi_{\mathbf{Z}^{\nu}-V_{N}}^{k}, \phi_{V_{M}}^{\prime}\right) \\
& -H\left(\phi_{V_{N}-V_{M}}^{\min , k} \mid \phi_{\mathbf{Z}^{\nu}-V_{N}}^{k}, \phi_{V_{M}}^{\prime}\right) \geq 0 .
\end{aligned}
$$

Thus, $\phi^{\text {min }}$ is a ground state that does not coincide with $\phi^{(p)}$. This contradiction shows that $L_{b}\left(\mathbf{Z}^{\nu}-V_{N}, \phi_{V_{M}}^{\prime}\right)$ is bounded. Lemma 1 is proven.

By combining the partition functions $\Xi^{\phi^{1}, \phi^{\prime}}$ and $\Xi^{\phi^{2}, \phi^{\prime \prime}}$ we define a double partition function $\Xi^{\phi^{1}, \phi^{\prime}, \phi^{2}, \phi^{\prime \prime}}=\Xi^{\phi^{1}, \phi^{\prime}} \Xi^{\phi^{2}, \phi^{\prime \prime}}$ :

$$
\Xi^{\phi^{1}, \phi^{\prime}} \Xi^{\phi^{2}, \phi^{\prime \prime}}=\sum \exp \left(-\beta H\left(\phi_{V_{N}-V_{M}}^{3} \mid \phi^{1}, \phi^{\prime}\right)\right) \exp \left(-\beta H\left(\phi_{V_{N}-V_{M}}^{4} \mid \phi^{2}, \phi^{\prime \prime}\right)\right)
$$

where the summation is taken over all pairs of configurations $\phi_{V_{N}-V_{M}}^{3}$ and $\phi_{V_{N}-V_{M}}^{4}$.

In the same way by combining of partition functions $\Xi^{\phi^{2}, \phi^{\prime}}$ and $\Xi^{\phi^{1}, \phi^{\prime \prime}}$ we define a double partition function $\Xi^{\phi^{2}, \phi^{\prime}, \phi^{1}, \phi^{\prime \prime}}$. 
The statistical weight of a contour $\gamma$ will be defined by the formula:

$$
\begin{aligned}
w(\gamma)= & \exp \left(-\beta\left(H\left(\phi\left(\operatorname{supp}(\gamma) \mid \phi_{\mathbf{Z}^{\nu}-\operatorname{supp}(\gamma)}^{(p)}\right)\right)\right.\right. \\
& \left.\left.-H\left(\phi^{(p)}\left(\operatorname{supp}(\gamma) \mid \phi_{\mathbf{Z}^{\nu}-\operatorname{supp}(\gamma)}^{(p)}\right)\right)\right)\right) .
\end{aligned}
$$

The collection of contours $\left\{\gamma_{1}, \ldots, \gamma_{m}\right\}$ is said to be compatible set if there exists a configuration $\phi$ which contains this set of contours. The partition function $\Xi^{\phi^{1}, \phi^{\prime}}$ naturally admits the following expansion

$$
\Xi^{\phi^{1}, \phi^{\prime}}=\exp \left(-\beta H\left(\phi_{V_{N}-V_{M}}^{(p)} \mid \phi^{1}, \phi^{\prime}\right)\right) \sum w\left(\gamma_{1}\right) \cdots w\left(\gamma_{m}\right) G\left(\gamma_{1}, \ldots, \gamma_{m}\right)
$$

where the summation is taken over all non-ordered compatible collections of contours and the interaction factor $G\left(\gamma_{1}, \ldots, \gamma_{m}\right)$ appears due to those contours among $\gamma_{1}, \ldots, \gamma_{m}$ which have non-empty intersection with the boundary $V_{M} \cup \mathbf{Z}^{\nu}-V_{N}$.

The following generalization of the definition of compatibility allows us to represent a double partition function $\Xi^{\phi^{1}, \phi^{\prime}, \phi^{2}, \phi^{\prime \prime}}$ as an ordinary partition function.

A set of contours is called two-compatible provided any of its two parts coming from two Hamiltonians is compatible. In other words, in two-compatibility an intersection of supports of two contours coming from different partition functions is allowed.

If $\left\{\gamma_{1}, \ldots, \gamma_{m}\right\}$ is a two-compatible set of contours and $\bigcup_{i=1}^{m} \operatorname{supp}\left(\gamma_{i}\right) \subset V_{N}-$ $V_{M}$, then there exist two configurations $\phi^{3}$ and $\phi^{4}$ which contain this set of clusters.

The double partition function is

$$
\begin{aligned}
\Xi^{\phi^{1}, \phi^{\prime}, \phi^{2}, \phi^{\prime \prime}}= & \Xi^{\phi^{1}, \phi^{\prime}} \Xi^{\phi^{2}, \phi^{\prime \prime}} \\
= & \exp \left(-\beta H\left(\phi_{V_{N}-V_{M}}^{(p)} \mid \phi^{1}, \phi^{\prime}\right)\right) \exp \left(-\beta H\left(\phi_{V_{N}-V_{M}}^{(p)} \mid \phi^{2}, \phi^{\prime \prime}\right)\right) \\
& \times \sum w\left(\gamma_{1}\right) \cdots w\left(\gamma_{m}\right) G\left(\gamma_{1}, \ldots, \gamma_{m}\right)
\end{aligned}
$$

where the summation is taken over all non-ordered two-compatible collections of contours.

Let $w\left(\gamma_{1}\right) \cdots w\left(\gamma_{m}\right)$ be a term of the double partition function $\Xi^{\phi^{1}, \phi^{\prime}, \phi^{2}, \phi^{\prime \prime}}$. The connected components of the collection $\left\{\operatorname{supp}\left(\gamma_{1}\right), \ldots, \operatorname{supp}\left(\gamma_{m}\right)\right\}$ are the supports of two-contours. A two-contour $\Gamma$ is a pair $(\operatorname{supp}(\Gamma), \phi(\operatorname{supp}(\Gamma))$.

A two-contour $\Gamma=\left\{\gamma_{1}, \ldots, \gamma_{m}\right\}$ is said to be long iff the intersection of the set $\bigcup_{i=1}^{m} \operatorname{supp}\left(\gamma_{i}\right)$ with both $V_{M+L_{b}}$ and $\mathbf{Z}^{\nu}-V_{N-L_{b}}$ is non-empty. In other words, a long two-contour by supports of its contours connects the $L_{b}$ neighborhood of the boundary with the $L_{b}$ neighborhood of the cube $V_{M}$. A set of two-contours is called compatible provided the set of contours belonging to these two-contours is two-compatible. We define the partition function

$$
\begin{aligned}
\Xi^{\phi^{1}, \phi^{\prime}, \phi^{2}, \phi^{\prime \prime},(n . l .)}= & \exp \left(-\beta H\left(\phi_{V_{N}-V_{M}}^{(p)} \mid \phi^{1}, \phi^{\prime}\right)\right) \exp \left(-\beta H\left(\phi_{V_{N}-V_{M}}^{(p)} \mid \phi^{2}, \phi^{\prime \prime}\right)\right) \\
& \times \sum w\left(\gamma_{1}\right) \cdots w\left(\gamma_{m}\right) G\left(\gamma_{1}, \ldots, \gamma_{m}\right)
\end{aligned}
$$

where the summation is taken over all non-ordered compatible collections of not long two-contours. 
Lemma 2. Let $\lambda$ be a value such that the model (3) has a unique ground state $\phi^{(p)}$. There exists a value of the inverse temperature $\beta_{0}(\lambda)$ such that for all $\beta>\beta_{0}(\lambda)$ the following statement holds: for each fixed cube $V_{M}$, there exists a number $N_{0}=$ $N_{0}(M)$, which depends on $M$ only such that if $N>N_{0}$ then

$$
\frac{1}{2} \Xi^{\phi^{1}, \phi^{\prime}, \phi^{2}, \phi^{\prime \prime}}<\Xi^{\phi^{1}, \phi^{\prime}, \phi^{2}, \phi^{\prime \prime},(n . l .)} .
$$

Proof. Define a partition function $\Xi^{\phi^{1}, \phi^{\prime}, \phi^{2}, \phi^{\prime \prime},(l .)}$ as

$$
\Xi^{\phi^{1}, \phi^{\prime}, \phi^{2}, \phi^{\prime \prime},(l .)}=\Xi^{\phi^{1}, \phi^{\prime}, \phi^{2}, \phi^{\prime \prime}}-\Xi^{\phi^{1}, \phi^{\prime}, \phi^{2}, \phi^{\prime \prime},(n . l .)} .
$$

Equivalently,

$$
1=\frac{\Xi^{\phi^{1}, \phi^{\prime}, \phi^{2}, \phi^{\prime \prime},(n . l .)}}{\Xi^{\phi^{1}, \phi^{\prime}, \phi^{2}, \phi^{\prime \prime}}}+\frac{\Xi^{\phi^{1}, \phi^{\prime}, \phi^{2}, \phi^{\prime \prime},(l)}}{\Xi^{\phi^{1}, \phi^{\prime}, \phi^{2}, \phi^{\prime \prime}}} .
$$

In order to prove the inequality (9) we have to show that

$$
\frac{\Xi^{\phi^{1}, \phi^{\prime}, \phi^{2}, \phi^{\prime \prime},(l)}}{\Xi^{\phi^{1}, \phi^{\prime}, \phi^{2}, \phi^{\prime \prime}}}<\frac{1}{2} .
$$

In other words, we have to prove that the probability of the event that there exists at least one long two-contour connecting $V_{M}$ with $\mathbf{Z}^{\nu}-V_{N}$ is less than $1 / 2$. This fact is a straightforward consequence of the Peierls argument. Indeed, since the spin space is finite, due to the condition (2) and the Peierls argument the probability of a contour

$$
P(\gamma)<\exp \left(-\beta \tau_{0}|\gamma|\right)
$$

for some positive $\tau_{0}<\tau$ where $|\gamma|$ denotes the number of basic cubes of linear size 2 in the support of contour $\gamma$. By definitions, the support of any two-contour is the union of contour supports or contour supports sitting on other contour supports. Therefore, the event "a fixed cube of linear size 2 is not regular" is a union of three events: "this cube is not regular in the first ensemble" ( with partition function $\Xi^{\phi^{1}, \phi^{\prime}}$ ), "this cube is not regular in the second ensemble" ( with partition function $\Xi^{\phi^{2}, \phi^{\prime \prime}}$ ) and "this cube is not regular in the first and second ensembles". Thus, for sufficiently large $\beta$ the probability of this event is less than $\exp \left(-\beta \tau_{0}\right)+\exp \left(-\beta \tau_{0}\right)+$ $\exp \left(-\beta \tau_{0}\right) \exp \left(-\beta \tau_{0}\right)<\exp \left(-\beta \tau_{1}\right)$, where $\tau_{1}=\tau_{0} / 2$.

If in the Gibbs distribution (4) we pass to the relative energies with respect to the configuration with minimal energy $\phi_{V_{N}-V_{M}}^{\min , k}$ then by Lemma 1 the area $V_{N-L_{b}}-$ $V_{M+L_{b}}$ is the "pure" area of the unique ground state $\phi^{(p)}$ and in this area by the Peierls argument the inequality (10) holds. Due to the fact that a long contour "starts" inside the cube $V_{M+L_{b}}$ and "ends" outside $V_{N-L_{b}}$, its diameter exceeds $N-M-2 L_{b}$. Therefore, the probability of existence of at least one long contour is less than $\sum_{n>N-M-2 L_{b}}\left(M+L_{b}\right)^{\nu}(2 \nu)^{n} \exp \left(-\beta n \tau_{1}\right)<\exp \left(-\beta \tau_{2}\left(N-M-2 L_{b}\right)\right)$ for some positive constant $\tau_{2}$ if $\beta$ is sufficiently large. At any fixed $M$ for sufficiently large values of $N$ the last expression is less then $1 / 2$. Lemma 2 is proven. 
Partition functions including only not long two-contours satisfy the following key lemma which has a geometrically-combinatorial explanation. An analogous lemma in more complicated long-range interaction case was firstly used in Ref. 15.

\section{Lemma 3.}

$$
\Xi^{\phi^{1}, \phi^{\prime}, \phi^{2}, \phi^{\prime \prime},(n . l .)}=\Xi^{\phi^{2}, \phi^{\prime}, \phi^{1}, \phi^{\prime \prime},(n . l .)} .
$$

Proof. The summations in $\Xi^{\phi^{1}, \phi^{\prime \prime}, \phi^{2}, \phi^{\prime},(n . l .)}=\Xi^{\phi^{1}, \phi^{\prime}, \phi^{2}, \phi^{\prime \prime},(n . l .)}$ are taken over all non-long, non-ordered compatible collections of two-clusters. Consider an arbitrary

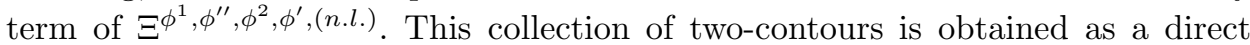
product of two collections of contours: a collection from $\Xi^{\phi^{1}, \phi^{\prime \prime}}$ and a collection from $\Xi^{\phi^{2}, \phi^{\prime}}$.

We say that a contour (two-contour) is a "root" contour ("root" two-contour), iff the intersection of its support with $V_{M+L_{b}} \cup\left(V_{N}-V_{N-L_{b}}\right)$ is not empty. For any not long root contour (two-contour) one the following four cases holds: its support has a nonempty intersection with

(1) $V_{N}-V_{N-L_{b}}$ and $\phi_{\mathbf{Z}^{\nu}-V_{N}}=\phi_{\mathbf{Z}^{\nu}-V_{N}}^{1}$;

(2) $V_{N}-V_{N-L_{b}}$ and $\phi_{\mathbf{Z}^{\nu}-V_{N}}=\phi_{\mathbf{Z}^{\nu}-V_{N}}^{2}$;

(3) $V_{M+L_{b}}$ and $\phi_{V_{M+L_{b}}}=\phi_{V_{M+L_{b}}}^{\prime}$;

(4) $V_{M+L_{b}}$ and $\phi_{V_{M+L_{b}}}=\phi_{V_{M+L_{b}}}^{\prime \prime}$.

In these cases we call the contour (two-contour) correspondingly a $\operatorname{root}^{1}$, $\operatorname{root}^{2}$, root' or root" contour (two-contour).

Now we put a one-to-one correspondence between the terms of these two double partition functions: for each pair of collections from $\Xi^{\phi^{1}, \phi^{\prime}}$ and $\Xi^{\phi^{2}, \phi^{\prime \prime}}$ we construct a pair of collections from $\Xi^{\phi^{2}, \phi^{\prime}}$ and $\Xi^{\phi^{1}, \phi^{\prime \prime}}$. First of all, for each root $^{1}$ contour $\gamma \in \Xi^{\phi^{1}, \phi^{\prime}}$ we construct the same $\operatorname{root}^{1}$ contour $\gamma \in \Xi^{\phi^{1}, \phi^{\prime \prime}}$, for each root' contour $\gamma \in \Xi^{\phi^{1}, \phi^{\prime}}$ we construct the same root' contour $\gamma \in \Xi^{\phi^{2}, \phi^{\prime}}$, for each $\operatorname{root}^{2}$ contour $\gamma \in \Xi^{\phi^{2}, \phi^{\prime \prime}}$ we construct the same $\operatorname{root}^{2}$ contour $\gamma \in \Xi^{\phi^{2}, \phi^{\prime}}$ and for each root" contour $\gamma \in \Xi^{\phi^{2}, \phi^{\prime \prime}}$ we construct the same root" contour $\gamma \in \Xi^{\phi^{1}, \phi^{\prime \prime}}$. After that, for all non-root contours $\gamma \in \Xi^{\phi^{1}, \phi^{\prime}}$ we construct the same non-root contours $\gamma \in \Xi^{\phi^{2}, \phi^{\prime}}$ and for all non-root contours $\gamma \in \Xi^{\phi^{2}, \phi^{\prime \prime}}$ we construct the same non-root contours $\gamma \in \Xi^{\phi^{1}, \phi^{\prime \prime}}$ if their supports do not intersect already constructed root contours. Finally, we move all newly constructed non-root contours $\gamma \in \Xi^{\phi^{2}, \phi^{\prime}}$ to $\Xi^{\phi^{1}, \phi^{\prime \prime}}$ if they have nonempty intersection with already constructed root contours and all newly constructed non-root contours $\gamma \in \Xi^{\phi^{1}, \phi^{\prime \prime}}$ to $\Xi^{\phi^{2}, \phi^{\prime}}$ if they have nonempty intersection with already constructed root contours.

Since all contours and two-contours are not long it can be readily shown that this one-to-one correspondence is well-defined. Lemma 3 is proven.

Now the inequality (6) with $c_{1}=1 / 2$ and $c_{2}=2$ readily follows from Lemma 3 and Lemma 2 for the partition function $\Xi^{\phi^{1}, \phi^{\prime}, \phi^{2}, \phi^{\prime \prime},(n . l .)}$ and $\Xi^{\phi^{2}, \phi^{\prime}, \phi^{1}, \phi^{\prime \prime},(n . l .)}$. Finally, Theorem 4, and therefore Theorem 3 is proven. 


\section{Conclusions}

The present proof of Theorem 3 gives a simple justification of the uniqueness phenomenon. The probability of the event that two contours connect a fixed cube $V_{M}$ with the boundary conditions goes to zero when volume $V_{N}$ increases. Therefore, the dependence on the boundary conditions naturally disappears in the limit.

\section{Acknowledgments}

The author thanks the referee for valuable suggestions.

\section{References}

1. S. A. Pirogov and Ya G. Sinai, Teoret. Mat. Fiz. 25, 358 (1975).

2. S. A. Pirogov and Ya G. Sinai, Teoret. Mat. Fiz. 26, 61 (1976).

3. Ya G. Sinai, Theory of Phase Transitions: Rigorous Results (Pergamon Press, Oxford, 1982).

4. R. Peierls, Proc. Cambridge Phil. Soc. 32, 477 (1936).

5. M. Zahradnik, Commun. Math. Phys. 93, 559 (1984).

6. R. L. Dobrushin and E. A. Pecherski, Colloquia Mathematica Societatis Janos Bolyai, Vol. 27(1) (North-Holland, Amsterdam, New York, 1981), p. 223.

7. S. B. Shlosman, Teor. Mat. Fiz. 66, 430 (1986).

8. D. G. Martirosyan, Teoret. Mat. Fiz. 63, 280 (1985).

9. J. L. Lebowitz and A. E. Mazel, Commun. Math. Phys. 189, 311 (1997).

10. J. Bricmont, J. L. Lebowitz and C. Pfister, J. Stat. Phys. 21, 573 (1979).

11. J. Van den Berg, Commun. Math. Phys. 152, 161 (1993).

12. J. Van den Berg and C. Maes, Ann. Prob. 22, 749 (1994).

13. H.-O. Georgii, Gibbs Measures and Phase Transitions (Walter de Gruyter, Berlin, 1988).

14. A. A. Kerimov, J. Phys. A 35, 5365 (2002).

15. A. A. Kerimov, J. Stat. Phys. 52, 69 (1993). 